TP Periodica Polytechnica Electrical Engineering and Computer Science

61(1), pp. 69-76, 2017

DOI: 10.3311/PPee. 10207

Creative Commons Attribution (i)

RESEARCH ARTICLE

\section{Performance Comparison of Multi Design Method and Meta-Heuristic Methods for Optimal Preliminary Design of Core-Form Power Transformers}

\author{
Tamás Orosz ${ }^{1 *}$, Bence Borbély ${ }^{1}, Z$ Zoltán Ádám Tamus ${ }^{1}$
}

Received 24 October 2016; accepted after revision 06 December 2016

\begin{abstract}
Large power transformers are regarded as crucial and expensive assets in power systems. Due to the competing global market, to make a good and competing power transformer design, a non-linear optimization problem should be solved in a very short time in the preliminary design stage. The paper shows and compares the performance of four different methods to solve this problem for three phase core type power transformers. The first algorithm is a novel meta-heuristic technique which combines the geometric programming with the method of branch and bound. Then this conventional multi design method is solved by a simple iterative technique and two novel evolutionary algorithms to enhance the convergence speed. One of these algorithms is the particle swarm optimization technique which is used by many other researchers and the grey wolf optimization algorithm which is a new method in this optimization sub-problem. An example design on an 80 MVA, three phase core type power transformer using these four methods is presented and its performances are analyzed. The results demonstrate that the grey wolf optimization is a good alternative for this optimization problem.
\end{abstract}

\section{Keywords}

evolutionary algorithms, mathematical optimization, power transformers, geometric programming, electrical machine design

\footnotetext{
${ }^{1}$ Department of Electrical Power Engineering, Faculty of Electrical Engineering and Informatics, Budapest University of Technology and Economics Egry J. u. 18, H-1111, Budapest, Hungary

* Corresponding author, e-mail: tamas.orosz@vet.bme.hu
}

\section{Introduction}

Making a good and competing power transformer design is a non-linear optimization problem. In an electrical machine the different electrodynamic, thermodynamic and mechanic fields interplay simultaneously. Therefore a lot of technical and economic factors need to be harmonized in the design process. Moreover by the large number of the optimized parameters this task belongs to the branch of the most general mathematical optimization problems $[1,2]$. Because of the complexity of this task the transformer design process is split up two main different stages in practice (Fig. 1): the preliminary and the final design stages [3-7].

The subject of this paper is only the preliminary design stage. Here the main challenging task is to find the key performance parameters of a cost optimal solution in a limited time to give a decent answer for a price quotation request. Generally, the main objective of the large electrical machine design is to minimize the total cost of ownership (TCO) by reducing the lifetime cost of the power losses and the manufacturing costs. Kapp mentioned this optimization problem in his book [8] at the beginning of the last century and showed a simple analytical approach to determine the key design parameters of the cost optimal transformer design. Since then a wide range of analytical and numerical method based algorithms have been proposed in the literature, from the simple approximative solutions [9] to the solvers which use the recent achievements of computational mathematics (e.g. neural networks, Monte Carlo methods, genetic algorithms, etc.) [9-11]. This shows that transformer design optimization is an active research field nowadays.

The focus of this paper - a geometric programming (GP) based meta-heuristic design optimization method - has been presented in the previous article of the authors [12]. This Geometrec Programming based novel solution has compared to three multi design transformer methods (MDM) $[2,13]$.

These three MDM subvariants which are examined in this paper, contain the same calculation methods as shown in [12] regarding meta-heuristic optimization, however, different optimum search algorithms are implemented (brute force search, particle swarm optimization (PSO) and gray wolf optimization (GWO)) to search the optimal design. The MDM method with 
a simple brute force search or combined with several genetic, evolutionary algorithms like PSO can be found in the literature $[2,9,14,15]$, but the GWO algorithm has not been used previously for this transformer design optimization problem.

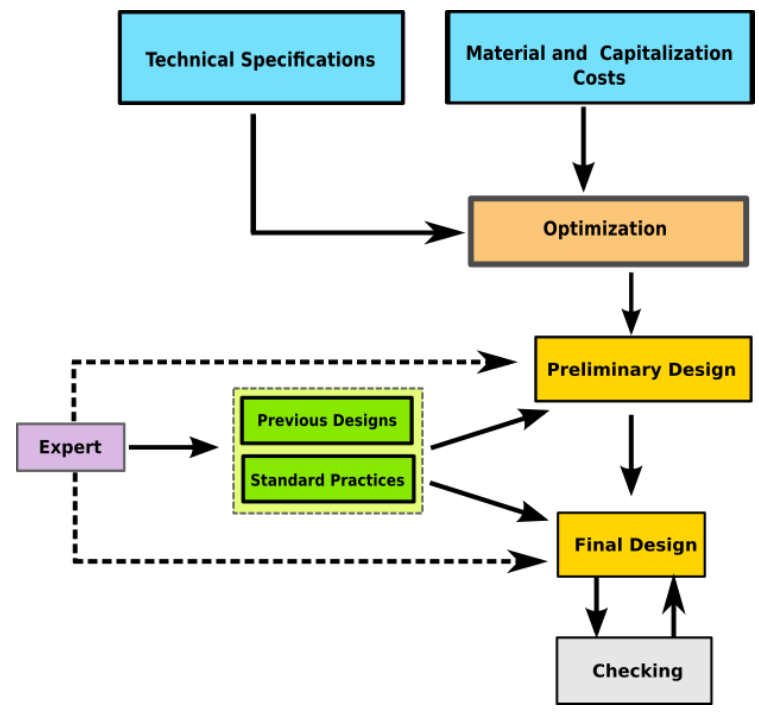

Fig. 1 Stages of a transformer design process [16]

The paper is organized in the following way. The first section gives a brief overview of the main design formulas of the realized transformer model. After that, in the second section the four examined optimum search models are shown, and finally in the Results and Discussion section, the performance of these four transformer design algorithms are discussed and compared in a real world transformer optimization task.

\section{Design formulas for the preliminary design optimization}

This section describes briefly the applied design formulas of a three phase core type power transformer optimization. This design process is the same as the geometric programming based algorithm [12, 17]. At this rough preliminary design stage the transformer is modelled by the active part (the core and windings) because its mass and dimensions determine the total cost of the equipment (Table 1). The generally used two winding model extended with a regulating winding and the dimensions of the transformer tank, which are already capable to take into consideration the limitations of the outer dimensions and the mass of the tank. By the sake of simplicity, in the examined models the high voltage winding is regulated with an on-load tap changer. The GP based algorithms take every performance parameter into consideration with a separate variable (Table 1) in the constraint equalities and inequalities which are formulated in a special posynomial and monomial format [18]. In contrast to the MDM based algorithms - which use the design formulas in their classical form - the new method contains and varies only the independent variables of this optimization problem.

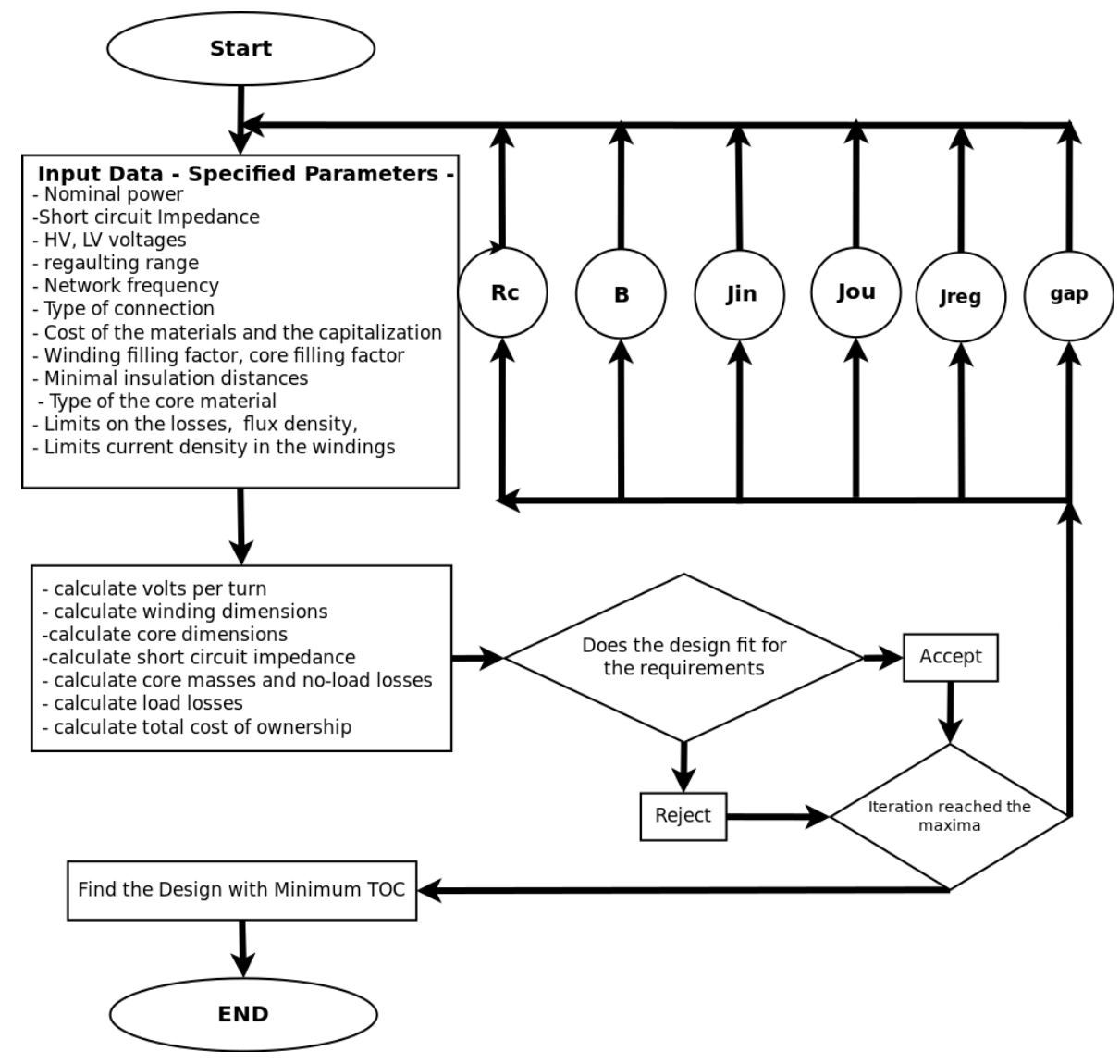

Fig. 2 The figure shows the steps of the realized MDM algorithm. 
Table 1 List of the transformer model's performance parameters to be optimized.

\begin{tabular}{|c|c|c|c|}
\hline \multicolumn{4}{|c|}{ Independent variables } \\
\hline \multicolumn{2}{|l|}{ Quantity } & Dimension & Variable \\
\hline \multicolumn{2}{|l|}{ Core diameter } & $\mathrm{mm}$ & $D_{c}$ \\
\hline \multicolumn{2}{|c|}{ Flux density in the core } & $\mathrm{T}$ & $B$ \\
\hline \multicolumn{2}{|c|}{ Main insulation distance } & $\mathrm{mm}$ & $g$ \\
\hline \multirow{3}{*}{ Current density } & secondary & $\frac{\mathrm{A}}{\mathrm{mm}^{2}}$ & $j_{s}$ \\
\hline & primary & $\frac{\mathrm{A}}{\mathrm{mm}^{2}}$ & $j_{p}$ \\
\hline & regulating & $\frac{\mathrm{A}}{\mathrm{mm}^{2}}$ & $j_{r}$ \\
\hline Winding height & secondary & $\mathrm{mm}$ & $h_{s}$ \\
\hline \multicolumn{4}{|l|}{ Dependent variables } \\
\hline \multicolumn{2}{|l|}{ Load Loss } & $\mathrm{kW}$ & $P_{l l}$ \\
\hline \multicolumn{2}{|l|}{ No Load Loss } & $\mathrm{kW}$ & $P_{n l l}$ \\
\hline \multicolumn{2}{|c|}{ Width of the working window } & $\mathrm{mm}$ & $s$ \\
\hline \multicolumn{2}{|l|}{ Core mass } & $\mathrm{t}$ & $M_{c}$ \\
\hline \multicolumn{2}{|l|}{ Tank length } & $\mathrm{mm}$ & $L$ \\
\hline \multicolumn{2}{|l|}{ Tank width } & $\mathrm{mm}$ & $W$ \\
\hline \multicolumn{2}{|l|}{ Tank height } & $\mathrm{mm}$ & $H$ \\
\hline \multirow{3}{*}{ Winding thickness } & secondary & $\mathrm{mm}$ & $t_{s}$ \\
\hline & primary & $\mathrm{mm}$ & $t_{p}$ \\
\hline & regulating & $\mathrm{mm}$ & $t_{r}$ \\
\hline \multirow{3}{*}{ Mean radius } & secondary & $\mathrm{mm}$ & $r_{s}$ \\
\hline & primary & $\mathrm{mm}$ & $r_{p}$ \\
\hline & regulating & $\mathrm{mm}$ & $r_{r}$ \\
\hline \multicolumn{4}{|c|}{ Abstract variables for short-circuit impedance calculation } \\
\hline \multicolumn{4}{|l|}{$\mathrm{a}, \mathrm{b}, \mathrm{c}, \mathrm{d}, \mathrm{e}, \mathrm{f}$} \\
\hline
\end{tabular}

\subsection{Objective function}

The objective function in each examined transformer optimization algorithm is based on the capitalized cost of the transformer. This function contains the manufacturing cost of the active part and the cost of the calculated losses:

$$
f_{0}=K_{1} \cdot P_{n l l}+K_{2} \cdot P_{l l}+\sum_{k=0}^{n} C_{k} \cdot M_{k},
$$

where $f_{0}$ is the TCO of the transformer's active part in $€$ and also the objective function of this optimization method. $K_{1}$ is the capitalized cost of the no-load loss and $K_{2}$ is the load loss capitalization cost in $€ / \mathrm{kW}$. $P_{n l l}$ is the no-load loss of the transformer in $\mathrm{kW}$ and $P_{l l}$ is the sum of the load losses generated in the transformers' active part in $\mathrm{kW}$ unit. $M_{k}$ is the mass of the part of the model which is represented by $k$ in $\mathrm{kg}$. $C_{k}$ represents the specific cost of the transformer part in $€ / \mathrm{kg}$.

\subsection{Turn voltage}

The turn voltage of the windings is calculated from the given power and the independent variables, the calculation can be formulated in the next form:

$$
P_{w l}=4.44 \lambda_{c} R_{c}^{2} \lambda_{\text {in }} f h_{i n} t_{\text {in }} j_{\text {in }}^{2}
$$

where $P_{w}$ is the power of a single wounded limb, $\lambda_{c}$ is the filling factor of the transformer's core.

\subsection{Core and no-load loss calculation}

Similarly to the meta-heuristic method in [12], in the case of a three phase three legged core, the core mass can be calculated by the following formulas:

$$
\begin{gathered}
M_{c}=M_{\text {leg }}+M_{\text {yoke }}+M_{\text {corner }}, \\
M_{\text {corner }}=R_{c}^{3} \cdot \lambda_{c} \cdot \pi \cdot \rho_{f e} \cdot \zeta, \\
M_{\text {column }}=R_{c}^{2} \cdot \lambda_{c} \cdot \pi \cdot \rho_{f e} \cdot\left(E I_{T O P}+E I_{B O T}+h_{\text {in }}\right), \\
M_{\text {yoke }}=R_{c}^{2} \cdot \lambda_{c} \cdot \pi \cdot \rho_{f e} \cdot\left(4 \cdot s+2 \cdot p_{d}+6 \cdot R_{c}\right),
\end{gathered}
$$

where $M_{\text {corner }}$ is the mass of the corners of the core, $M_{\text {leg }}$ is the mass of the leg, $M_{\text {yoke }}$ is the mass of the yoke. $\lambda_{c}$ is the filling factor of the core, it depends on the quality of the applied electrical steel and the construction technology. $\zeta$ is a technology dependent factor for the core volume calculation, $\rho_{f e}$ is the density of the electrical steel. $E I_{T O P}$ and $E I_{B O T}$ are the end insulation distances, between the bottom and the top of the yoke and the inner winding and $p_{d}$ is the phase insulation. The $h_{i n}$ winding is used as a reference height in the model as in the meta-heuristic method based optimization [12]. The height of the outer and the regulating windings are taken into consideration by a simple multiplication of one factor.

A loss function is fitted to the applied electrical steel [19] like in the case of the meta-heuristic method $[1,12,20]$ :

$$
P_{n l l}=M_{c} \cdot f_{b} \cdot p_{n l l}
$$

where, $f_{b}$ is the building factor which depends on the technology. The effect of the manufacturing technology and the building-factor whose typical value is 1.2 in the case of a rough calculation at the preliminary design stage.

\subsection{Winding mass and loss calculation}

The mass of a transformer winding is formulated in the next form:

$$
m_{k}=2 \pi \rho \lambda_{k}\left(R_{k}+t_{k}\right) t_{k} \alpha_{k} h_{i n}
$$

where $\rho$ is the copper density and $\lambda_{k}$ is the copper filling factor in the winding $k . \alpha_{k}$ is a constant multiplier, which takes the height of the winding into account. $R_{k}, t_{k}$ are the 
parameters of the examined winding, $h_{i n}$ is the height of the inner winding, which is usually the highest [21]. $\left(R_{k}+t_{k}\right)$ takes into consideration that

$$
P_{l l, k}=\sum \rho_{C u} m_{k} \cdot j_{k}(1+\kappa)
$$

where $\rho_{C u}$ is the copper resistance $\Omega \cdot m$ at $75^{\circ} \mathrm{C}, k$ represents the winding, $j_{k}$ is the current density in the $k$ th winding in $A / \mathrm{mm}^{2} . \kappa$ is an empirical constant which consider the optimal ratio $P_{A C} / P_{D C}$ of the losses $[1,22]$.

\subsection{Short Circuit Impedance (SCI)}

The SCI can be calculated by analytically in the following way:

$$
\operatorname{SCI}(\%)=\frac{2 \pi \mu_{0} f P_{w l}}{U_{T}^{2}(h+0.32 s)} \cdot\left(\frac{R_{i n} t_{i n}}{3}+\frac{R_{o u} t_{o u}}{3}+R_{m} g\right)
$$

where the $S C I$ is given in [\%]. The $U_{T}$ means the turn voltage, $P_{w l}$ the built-in power in a wounded limb. In this type of transformer core it holds that $P_{w l}=P_{\text {phase }}$ where $P_{\text {phase }}$ is the phase power of a transformer. $R_{m}$ is the mean diameter of the main gap of the transformer.

\section{Meta-heuristic method}

The modern interior-point method based GP solvers can be applied to solve the transformer design optimization task, which guarantees that the obtained solution is the global optimum [17]. Moreover, even large scale geometric programs can be solved extremely efficiently and reliably [18, 23]. However, the description of this branch of mathematical optimization methods is quite restrictive. The expressions of the equality and inequality constraints are prescribed to be special mathematical formulae called monomials (Eq. (11)) and posynomials (Eq. (12)). Here $c_{k}>0$ and the $\alpha$ parameters are real numbers. Every $x$ variable value has to be positive as well.

$$
\begin{gathered}
m(x)=c_{g} \cdot x_{1}^{\alpha_{1}} \cdot x_{2}^{\alpha_{2}} \cdot \ldots \cdot x_{n}^{\alpha_{n}} \\
p(x)=\sum_{k=1}^{K} c_{k} \cdot x_{1}^{\alpha_{1 k}} \cdot x_{2}^{\alpha_{2} k} \cdot \ldots \cdot x_{n}^{\alpha_{n} k}
\end{gathered}
$$

But as the authors have shown, this method cannot been used in the case of core-form transformers, where the short circuit impedance is required $[12,20]$. To solve this problem a meta-heuristic algorithm was created along the lines of Branch and Bound search (BB). The BB executes a binary search in sense that the solution space is divided into several subspaces, which can be described in appropriate convex terms $[12,20]$. The $\mathrm{BB}$ can produce large number of sub-cases, but the $\mathrm{BB}$ is usually quite efficient in the practice because it employs advanced techniques to remove certain parts of the search tree before evaluation. The general solution for core-form power transformers are deeply described in [12].

\section{Evolutionary algorithms}

In the optimization process, besides the brute force technique and geometric programming, two different meta-heuristic procedures were considered as well, namely particle swam optimization (PSO) and grey wolf optimization (GWO). Such computational methods, regardless the problem on which they are applied, do not guarantee the globally optimal solution to be found. In return, they provide the ability of much faster scanning of large search spaces, which are not feasible to be sampled completely and finely enough. On the other hand, since they do not use the gradient of the problem as opposed to the classic optimization methods, they do not require the problem to be differentiable.

\subsection{Particle swarm optimization}

Particle swarm optimization, first proposed by Ebenhard and Kennedy [24], was intended to model social behaviour, e.g. in a bird flock. The main idea is based on a so called swarm, an initial population of candidate solutions, which are moved in the search space during optimization, i.e., during the search for the global extrema of a function $f: \mathbb{R}^{n} \rightarrow \mathbb{R}$ called the cost function in a search space $S \subset \mathbb{R}^{n}$. Without the loss of generality let us confine ourselves to a minimization problem, when the goal is to find $\mathbf{a} \in S$ such that $\forall \mathbf{b} \in S, f(\mathbf{a}) \leq f(\mathbf{b})$. Let $N$ be the number of particles (candidate solutions) in the swarm and define $\mathbf{x}_{i}$ and $\mathbf{v}_{i}$ to be the position and the velocity of a particle in the search space, respectively, for $i=1, \ldots, N$. Moreover, let $\mathbf{p}_{i}$ denote the best known position of the $i$ th particle and $\mathbf{g}$ the best known position of all the particles in the swarm. Let us introduce three freely chosen scalar values, $\omega, \varphi_{p}$ and $\varphi_{g}$ controlling the efficiency and behaviour of the optimization process. On assigning an appropriate value to these variables see [25] and [26]. The algorithm consists of the following simple steps:

1. Initialize the position $\mathbf{x}_{i}$ of every particle in the swarm with a uniformly distributed random vector from the search space $S$,

2. Initialize $\mathbf{p}_{i} \leftarrow \mathbf{x}_{i}, i=1, \ldots, N$ and then $\mathbf{g}$ with the $\mathbf{p}_{i}$ of the least cost,

3. Initialize the components of every $\mathbf{v}_{i}$ with a uniformly distributed random values from $\left\{\mathbf{v}_{i, j} \mid \mathbf{b}_{l, j} \leq \mathbf{v}_{i, j} \leq \mathbf{b}_{u, j}\right.$, $j=1, \ldots, N\}$, where $\mathbf{b}_{l}$ and $\mathbf{b}_{u}$ are the lower and upper bounds of the search space,

4. Do until iteration $M$ is reached or termination criterion is met:

- For every particle $i=1, \ldots, N$, do:

- For every dimensions $d=1, \ldots, n$, do:

* Pick random numbers $r_{p}, r_{g}$ from $[0,1]$,

* Update particle velocity: $\mathbf{x}_{i} \leftarrow \mathbf{x}_{i}+\mathbf{v}_{i}$,

- Update position $\mathbf{x}_{i}$ of each particle by $\mathbf{x}_{i} \leftarrow \mathbf{x}_{i}+\mathbf{v}_{i}$,

- Update $\mathbf{p}_{i}$ and $\mathbf{g}$ such that $\mathbf{v}_{i, d} \leftarrow \omega \mathbf{v}_{i, d}+\varphi_{p} r_{p}$ $\left(\mathbf{p}_{i, d}-\mathbf{x}_{i, d}\right)+\varphi_{g} r_{g}\left(\mathbf{g}_{d}-\mathbf{x}_{i, d}\right)$, 


$$
\begin{aligned}
& f\left(\mathbf{x}_{i}\right)<f\left(\mathbf{p}_{i}\right) \quad \mathbf{p}_{i} \leftarrow \mathbf{x}_{i}, \\
& f\left(\mathbf{p}_{i}\right)<f(\mathbf{g}) \quad \mathbf{g} \leftarrow \mathbf{p}_{i},
\end{aligned}
$$

5. The most optimal solution found is contained in $\mathbf{g}$.

\subsection{Grey wolf optimization}

The grey wolf optimization, proposed by Mirjalili et. al. [27], mimics the social characteristics in grey wolf packs and simulates their hunting behavior. Similarly to PSO, GWO is a swarm-based meta-heuristic. The population is divided into four groups, referred to as alpha $(\alpha)$, beta $(\beta)$, delta $(\delta)$ and omega $(\omega)$, respectively. The three best instances having the minimal fitness values are alpha, beta and delta in ascending order. The remaining instances are the omega, which during the optimization are re-positioned in the search space according to the $\alpha, \beta$ and $\delta$. Let $X^{(i)}$ denote the position of a grey wolf in the $i$ th iteration, $X_{p}^{(i)}$ the position of the prey in the $i$ th iteration and the operator - element-wise multiplication between vectors of the same size.

The mathematical model of the encircling, a notable part in the hunting process, can be described by the following formulas

$$
\begin{gathered}
\mathbf{D}=\left|\mathbf{C} \cdot \mathbf{X}_{p}^{(i)}-\mathbf{X}^{(i)}\right|, \\
\mathbf{X}^{(i+1)}=\mathbf{X}_{p}^{(i)}-\mathbf{A} \cdot \mathbf{D},
\end{gathered}
$$

where $\mathbf{A}$ and $\mathbf{C}$ are coefficient vectors, given by

$$
\begin{gathered}
A=2 a \cdot r_{1}-a, \\
C=2 r_{2},
\end{gathered}
$$

where $r_{1}, r_{2}$ are random vectors with components from $[0,1]$ and $a$ varies linearly from 2 to 0 according to iteration.

Since the position $\mathbf{X}_{p}$ of the prey, the global optimum, is not known, the $\alpha, \beta$ and $\delta$ wolves are assumed to have a better knowledge about it. Consequently, the position of a wolf is updated according to the following equation

$$
\mathbf{X}^{(i+1)}=\frac{1}{3} \sum_{j=1}^{3} \mathbf{X}_{j},
$$

where

$$
\begin{aligned}
& \mathbf{X}_{1}=\mathbf{X}_{\alpha}-\mathbf{A}_{1} \cdot \mathbf{D}_{\alpha}, \\
& \mathbf{X}_{2}=\mathbf{X}_{\beta}-\mathbf{A}_{2} \cdot \mathbf{D}_{\beta}, \\
& \mathbf{X}_{3}=\mathbf{X}_{\delta}-\mathbf{A}_{3} \cdot \mathbf{D}_{\delta},
\end{aligned}
$$

and

$$
\begin{aligned}
& \mathbf{D}_{\alpha}=\left|\mathbf{C}_{1} \cdot \mathbf{X}_{\alpha}-\mathbf{X}\right|, \\
& \mathbf{D}_{\beta}=\left|\mathbf{C}_{2} \cdot \mathbf{X}_{\beta}-\mathbf{X}\right|,
\end{aligned}
$$

$$
\mathbf{D}_{\delta}=\left|\mathbf{C}_{3} \cdot \mathbf{X}_{\delta}-\mathbf{X}\right| .
$$

The general steps of the GWO algorithm are the following:

1. Initialize the pack of wolves randomly within the bounds of the search space,

2. Do until iteration $M$ is reached or termination criterion is met:

(a) Calculate the value of the objective function for all the wolves in the population,

(b) Chose the three instances with the best objective value to be the $\alpha, \beta$ and the $\delta$, respectively,

(c) Update the position of the $\omega$ wolves according to Eq. (17),

(d) Update $a, r_{1}$ and $r_{2}$,

3. The position of the $\alpha$ wolf is the best approximated optimum.

\section{Results and Discussion}

\subsection{Test Case Transformer Specification}

The above mentioned four optimization methods have been compared regarding a three-phase, 80 MVA power transformer. For this comparison two different sets of capitalisation factors have been selected $\left(k_{1}, k_{2}\right)$ from [28]. Thereforethe value of the no-load loss capitalization is $k_{1}=7000$, the value of the load loss capitalization factor in $€ / \mathrm{kW} \quad k_{2}=1000 € / \mathrm{kW}$. The detailed data-sheet with all of the required electrical and mechanical parameters are presented in Table 2.

For the sake of simplicity, the transformer cooling was chosen to be ONAN and the ambient temperature was specified to $40{ }^{\circ} \mathrm{C}$. The allowed winding oil temperature rise was defined to $\Theta_{w o}=65 \mathrm{~K}$, according to the IEC-60076 standard [29]. Therefore a winding current density limit was set to $3 \mathrm{~A} / \mathrm{mm}^{2}$ in the main windings and $3.5 \mathrm{~A} / \mathrm{mm}^{2}$ in the regulating winding. The windings were modeled by their copper filling factors which factors were based on the technology and the manufacturing experience.

The applied core material in this case was a TRAN-COR $\mathrm{H} 1$ grade electrical steel [19]. The maximum of the flux density was limited to $1.7 \mathrm{~T}$ considering the saturation of the core material and the over-voltages in the power grid.

The minimal insulation distances were chosen by a priory empirical rules $[1,21,30,31]$. These methods were based on the lightning impulse test and $\mathrm{AC}$ test requirements.

\subsection{Discussion of the Results}

Table 3 summarizes the optimal design variables which were calculated by the four different optimization methods. The value of the objective functions and the computational resource of the different methods can be compared.

One calculation step in the different algorithms has a similar calculation effort. The MDM method which iterates over the given set of variables is the slowest from the compared 
methods $\left(O\left(n^{6}\right)\right)$. Fig. 3 shows the shape of the optimal TCO in the function of the turn voltage, and illustrates the large amount of possible solutions. For this graph $n=200000$ is made, but as the results of Table 3 shows this algorithm haven't found the global optimum as the meta-heuristic method. Because of the improper discretization of the variable spaces.

Table 2 List of the transformer model parameters.

\begin{tabular}{|c|c|c|c|}
\hline \multicolumn{2}{|l|}{ Parameter } & Dimension & Value \\
\hline \multicolumn{2}{|c|}{ Nominal power } & MVA & 80 \\
\hline \multicolumn{2}{|l|}{ Frequency } & $\mathrm{Hz}$ & 50 \\
\hline \multicolumn{2}{|c|}{ Connection group } & & YNd11 \\
\hline \multicolumn{2}{|c|}{ Number of phases } & $\#$ & 3 \\
\hline \multicolumn{2}{|c|}{ Short circuit impedance } & $\%$ & 16.5 \\
\hline \multicolumn{2}{|l|}{ Main gap } & $\mathrm{mm}$ & 71 \\
\hline \multicolumn{2}{|c|}{ Sum of the end insulation distance } & $\mathrm{mm}$ & 370 \\
\hline \multicolumn{2}{|c|}{ LV side distance } & $\mathrm{mm}$ & 155 \\
\hline \multicolumn{2}{|c|}{ HV side distance } & $\mathrm{mm}$ & 330 \\
\hline \multicolumn{2}{|c|}{ Phase distance } & $\mathrm{mm}$ & 71 \\
\hline \multicolumn{2}{|c|}{ Core-Tank insulation top } & $\mathrm{mm}$ & 60 \\
\hline \multicolumn{2}{|c|}{ Core-Tank insulation bottom } & $\mathrm{mm}$ & 90 \\
\hline \multirow{5}{*}{ Core } & Number of legs & \# & 3 \\
\hline & Flux density limit & $\mathrm{T}$ & 1.73 \\
\hline & Filling Factor & $\%$ & 90 \\
\hline & Material Type & & $27 \mathrm{H} 074$ \\
\hline & Material Price & $€ / \mathrm{kg}$ & 3 \\
\hline \multirow{5}{*}{$\begin{array}{l}\text { Low Voltage } \\
\text { Winding }\end{array}$} & Line Voltage & $\mathrm{kV}$ & 33 \\
\hline & Phase Voltage & $\mathrm{kV}$ & 19.05 \\
\hline & Phase Current & A & 1399.64 \\
\hline & Copper filling factor & $\%$ & 70 \\
\hline & $\begin{array}{l}\text { Material and } \\
\text { manufacturing price }\end{array}$ & $€ / \mathrm{kg}$ & 8 \\
\hline \multirow{7}{*}{$\begin{array}{l}\text { High Voltage } \\
\text { Winding }\end{array}$} & Line Voltage & $\mathrm{kV}$ & 225 \\
\hline & Phase Voltage & $\mathrm{kV}$ & 129.9 \\
\hline & Phase Current & A & 205.28 \\
\hline & BIL & $\mathrm{kV}$ & 1050 \\
\hline & $\mathrm{AC}$ & $\mathrm{kV}$ & 460 \\
\hline & Copper filling factor & $\%$ & 50 \\
\hline & $\begin{array}{l}\text { Material and } \\
\text { manufacturing price }\end{array}$ & $€ / \mathrm{kg}$ & 7 \\
\hline \multirow{4}{*}{$\begin{array}{l}\text { Regulating } \\
\text { Winding }\end{array}$} & Regulating range & $\%$ & 15 \\
\hline & Insulation & & Fully insulated \\
\hline & Regulated winding & & High voltage \\
\hline & Filling factor & $\%$ & 70 \\
\hline
\end{tabular}

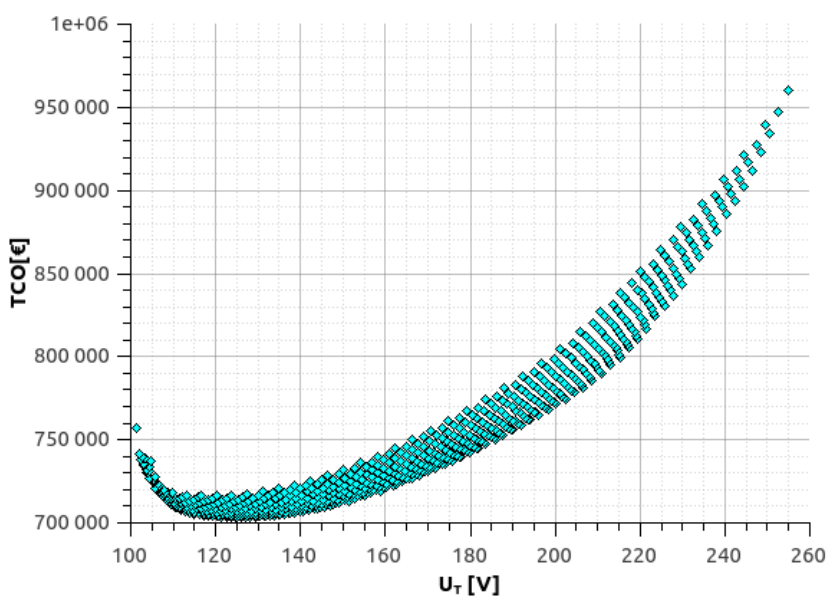

Fig. 3 The optimal value of the TCO in the function of the turn voltages in the case of the multi design optimization method.

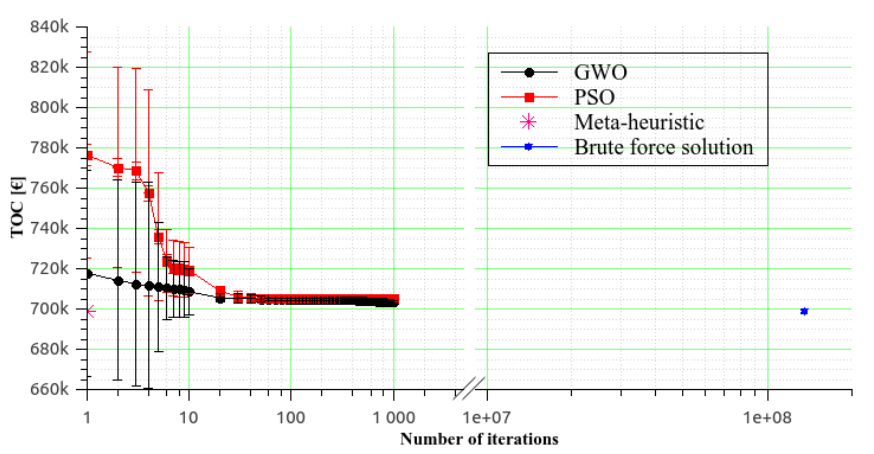

Fig. 4 Average performance of the applied PSO and the GWO algorithms.

Table 3 and Fig. 4 shows that the two evolutionary algorithm improves the convergence of the MDM significantly, according to the literature $[9,32]$. Fig. 4 shows that less than 50 iterations are enough by the presented PSO or GWO based methods to achieve the accuracy of the simple iteration based MDM method. Fig. 4 compares the average convergence rate of the two evolutionary algorithm based methods. This average performance representation is made of 50 runs with linear topology, in both cases. The GWO algorithm shows a greater convergence rate than the PSO algorithm after few iterations, but after 100 iterations no significant difference can be seen between the average performances (Fig. 4).

In this case the meta-heuristic method has found the optimal solution after only one iteration. Therefore, in this application the meta-heuristic search is the fastest from the four compared algorithms. But it should be noted that the convergence speed of the meta-heuristic method is depends on the value of the required SCI. As the previous study showed [12] if the optimal value of the SCI is lesser or equal to the requirement, only one iteration can be enough otherwise it can be complicated. The number of the iterations in this algorithm depends on the distance between the optimal value of the impedance and the 
Table 3 Values of the MDM method independent variables in the compared optimization methods.

\begin{tabular}{|c|c|c|c|c|c|}
\hline & & MDM & PSO & GWO & meta-heuristic \\
\hline core diameter & $\mathrm{mm}$ & 750 & 747.45 & 753 & 746 \\
\hline flux density & $\mathrm{T}$ & 1.42 & 1.42 & 1.41 & 1.4 \\
\hline core mass & $\mathrm{t}$ & 43.4 & 43.50 & 43.61 & 42.9 \\
\hline core loss & $\mathrm{kW}$ & 27.3 & 27.97 & 27.25 & 27 \\
\hline turn voltage & $\mathrm{V}$ & 124.8 & 126.77 & 125.58 & 123.6 \\
\hline inner winding height & $\mathrm{mm}$ & 1760 & 1712.55 & 1737.31 & 1766 \\
\hline \multicolumn{6}{|l|}{ Current density } \\
\hline inner winding & $\mathrm{A} / \mathrm{mm} 2$ & 2.37 & 2.74 & 2.40 & 2.0 \\
\hline outer winding & $\mathrm{A} / \mathrm{mm} 2$ & 1.98 & 1.80 & 2.0 & 2.0 \\
\hline regulating winding & $\mathrm{A} / \mathrm{mm} 2$ & 3.25 & 2.80 & 3.24 & 3.0 \\
\hline \multicolumn{6}{|l|}{ Totals } \\
\hline load loss & $\mathrm{kW}$ & 244.5 & 245.17 & 246.43 & 244.1 \\
\hline main gap & $\mathrm{mm}$ & 71 & 71 & 71 & 71 \\
\hline copper mass & $\mathrm{t}$ & 18.73 & 18.73 & 18.41 & 18.1 \\
\hline objective function & $€$ & 703437 & 708056 & 703328 & 698846 \\
\hline
\end{tabular}

required SCI. If the optimal value of the SCI is lower than the required, the algorithm finds the global optimum in the first step, but if the impedance is greater, about 100 iterations are enough to find an accurate solution. The value of the objective function is significantly better in this case than in that of the MDM method. The difference between the objectives is lesser than $1 \%$, but the difference between the current densities is about $20 \%$ because of the non-linearity of the transformer design problem. In contrast to the PSO and GWO algorithms, no significant difference can be shown.

\section{Conclusion}

The paper presents and compares the performance of four different design optimization methods in the case of core-form power transformers. The MDM method can be implemented and applied easily, but it has a large computational requirement, and does not guarantee to find the global optimum of the transformer optimization problem. The difference between the relative values of the object functions are relatively small, but the difference between the computational cost is significant. The meta-heuristic algorithm which combines the geometric programming with the method of branch and bound gives more accurate results in much shorter time than any other examined method. The gray wolf optimization algorithm was applied successfully for the transformer optimization problem, and has a better performance for low iterations than the particle swarm optimization. By the application of these evolutionary strategies the performance of the MDM method can be improved significantly and can reach about the accuracy of the metaheuristic method.

\section{References}

[1] Del Vecchio, R. M., Poulin, B., Feghali, P. T., Shah, D. M., Ahuja,R. "Transformer Design Principles: With Applications to Core-Form Power Transformers." CRC Press, 2010.

[2] Georgilakis, P. S. "Spotlight on modern transformer design." SpringerVerlag, London. 2009. https://doi.org/10.1007/978-1-84882-667-0

[3] Abetti, P., Cuthbertson, W., Williams, S. "Philosophy of applying digital computers to the design of electric apparatus." Transactions of the American Institute of Electrical Engineers, Part I: Communication and Electronics. 77(3), pp. 367-379. 1958. https://doi.org/10.1109/tce.1958.6372814

[4] Adly, A. A. "A specifications-oriented initial design methodology for power transformers." Energy Systems. pp. 1-12. 2016. https://doi.org/10.1007/s12667-016-0197-5

[5] Orosz, T., Vajda, I. "Design optimization with geometric programming for core type large power transformers." Electrical, Control and Communication Engineering. 6(1), pp. 13-18. 2014. https://doi.org/10.2478/ecce-2014-0012

[6] Parvaneh, H., Sedighizadeh, M. "Optimum designing of a Transformer Considering Lay out Constraints by Penalty-Based Method using Hybrid Big Bang-Big Crunch Approach." International Journal of Civil, Mechanical and Energy Science. 2(1), pp. 18-24. 2016.

[7] Andersen, O. W. "Optimized design of electric power equipment." IEEE Computer Applications in Power. 4(1), pp. 11-15, 1991. https://doi.org/10.1109/67.65030

[8] Kapp, G. "Transformatoren für Wechselstrom und Drehstrom." (Transformers for Single and MultiphaseCurrents: A Treatise on Their Theory, Construction, and Use). Nabu Press, Charleston SC, United States, 2012. (in German)

[9] Khatri, A., Malik, H., Rahi, O. P. "Optimal design of power transformer using genetic algorithm." In: 2012 International Conference on Communication Systems and Network Technologies, CSNT-2012, MIR Labs, Chapter Building, Rajkot, Gujarat, India, May 11-13, 2012. pp. 830-833. https://doi.org/10.1109/CSNT.2012.180 
[10] Amoiralis, E. I., Tsili, M. A., Georgilakis, P. S. "The state of the art in engineering methods for transformer design and optimization: a survey." Journal of Optoelectronics and Advanced Materials. 10(5), pp. 1149-1158. 2008

[11] Mehta, H., Patel, R. "Optimal Design of Transformer Using Tournament Selection Based Elitist Genetic Algorithms." Indian Journal of Science andTechnology. 8(12), p. 9. 2015. https://doi.org/10.17485/ijst\%2F2015\%2Fv8i12\%2F59187

[12] Orosz, T., Sleisz, A., Tamus, Z. A. "Metaheuristic Optimization Preliminary Design Process Of Core-Form Autotransformers." IEEE Transactions on Magnetics. 52(4), pp. 1-10. 2016. https://doi.org/10.1109/TMAG.2015.2496905

[13] Olivares-Galvan, J. C., Georgilakis, P. S., Escarela-Perez, R., CamperoLittlewood, E. "Optimal design of single-phaseshell-type distribution transformers based on a multiple design method validated by measurements." Electrical Engineering. 93(4), pp. 237-246. 2011. https://doi.org/10.1007/s00202-011-0211-9

[14] Amoiralis, E. I., Tsili, M. A., Kladas, A. G. "Transformer design and optimization: a literature survey." IEEE Transactionson on Power Delivery. 24(4), pp. 1999-2024. 2009. https://doi.org/10.1109/TPWRD.2009.2028763

[15] Qin, H., Kimball, J. W., Venayagamoorthy, G. K. "Particle swarm optimization of high-frequency transformer." In: 36th Annual Conference on IEEE Industrial Electronics Society, IECON 2010, Renaissance Hotel \& Spa Glendale, AZ, USA, Nov. 7-10, 2010. pp. 2914-2919. https://doi.org/10.1109/IECON.2010.5674932

[16] Orosz, T., Kleizer, G., Iváncsy, T., Tamus, Z. Á. „Comparison of methods for calculation of core-form power transformer's coretemperature rise." Periodica Polytechnica Electrical Engineering and Computer Science. 60(2), pp. 88-95. 2016. https://doi.org/10.3311/PPee.8893

[17] Jabr, R. A. "Application of geometric programming to transformer design." IEEE Transactions on Magnetics. 41(11), pp. 4261-4269. 2005. https://doi.org/10.1109/TMAG.2005.856921

[18] Boyd, S., Vandenberghe, L. "Convex optimization." Cambridge University Press, 2004.

[19] AK-Steel, "TRAN-COR® H-GRAIN oriented electrical steels." Jun 2013. URL: http://www.aksteel.com/markets_products/electrical.aspx

[20] Orosz, T., Sleisz, Á., Vajda, I. "Core-formtransformer design optimization with branch and bound search and geometric programming." In: 2014 55th International Scientific Conference on Power and Electrical Engineering of Riga Technical University (RTUCON), 2014. pp. 17-21. https://doi.org/10.1109/RTUCON.2014.6998194
[21] Karsai, K., Kerenyi, D., Kiss, L. "Large power transformers." Elsevier Science, New York, NY, 1987.

[22] Hurley, W. G., Gath, E., Breslin, J. G. "Optimizing the AC resistance of multilayer transformer windings with arbitrary current waveforms." IEEE Transactionson on Power Electronics. 15(2), pp. 369-376. 2000. https://doi.org/10.1109/63.838110

[23] Boyd, S., Kim, S.-J., Vandenberghe, L., Hassibi, A. "A tutorial on geometric programming." Optimization and Engineering. 8(1), pp. 67-127. 2007. https://doi.org/10.1007/s11081-007-9001-7

[24] Kennedy, J., Eberhart, R. "Particles warm optimization." In: Neural Networks, 1995. Proceedings., IEEE International Conference on, Perth, WA, 27 Nov.-1 Dec., 1995, pp. 1942-1948. https://doi.org/10.1109/ICNN.1995.488968

[25] Shi, Y., Eberhart, R. C. "Parameter selection in particle swarm optimization." In: EvolutionaryProgramming VII. (Porto, V. W., Saravanan, N., Waagen, D., Eiben, A. E. (eds.)), pp. 591-600. Springer, 1998. https://doi.org/10.1007/BFb0040810

[26] Trelea, I. C. "The particle swarm optimization algorithm: convergence analysis and parameter selection." Information Processing Letters. 85(6), pp. 317-325. 2003. https://doi.org/10.1016/S0020-0190(02)00447-7

[27] Mirjalili, S., Mirjalili, S. M., Lewis, A. "Grey wolf optimizer." Advances in Engineering Software. 6, pp. 46-61. 2014. https://doi.org/10.1016/j.advengsoft.2013.12.007

[28] Orosz, T., Sőrés, P., Raisz, D., Tamus, A. Z. "Analysis of the green power transition on optimal power transforme rdesigns." Periodica Polytechnica Electrical Engineering and Computer Science. 69(3), pp. 125-131. 2015. https://doi.org/10.3311/PPee.8583

[29] IEC Standard Power Transformers-Temperature Rise, Std. IEC Std 60 076-2, 1993.

[30] Ryan, H. M. "High voltage engineering and testing." IET, 2013.

[31] Kulkarni, S. V., Khaparde, S. "Transformer engineering: design and practice." CRC Press, 2004.

[32] Georgilakis, P. S., Amoiralis, E. I. "Distribution transformer cost evaluation methodology in corporating environmental cost." IET Generation, Transmission \& Distribution. 4(7), pp. 861-872. 2010. https://doi.org/10.1049/iet-gtd.2009.0638 